Internat. J. Math. \& Math. Sci.

Vol. 24, No. 11 (2000) 721-727

S0161171200005184

(C) Hindawi Publishing Corp.

\title{
STABILITY OF GENERALIZED ADDITIVE CAUCHY EQUATIONS
}

\author{
SOON-MO JUNG and KI-SUK LEE
}

(Received 9 May 2000)

ABSTRACT. A familiar functional equation $f(a x+b)=c f(x)$ will be solved in the class of functions $f: \mathbb{R} \rightarrow \mathbb{R}$. Applying this result we will investigate the Hyers-Ulam-Rassias stability problem of the generalized additive Cauchy equation

$$
f\left(a_{1} x_{1}+\cdots+a_{m} x_{m}+x_{0}\right)=\sum_{i=1}^{m} b_{i} f\left(a_{i 1} x_{1}+\cdots+a_{i m} x_{m}\right)
$$

in connection with the question of Rassias and Tabor.

Keywords and phrases. Generalized additive Cauchy equation, Hyers-Ulam-Rassias stability.

2000 Mathematics Subject Classification. Primary 39B22, 39872.

1. Introduction. The starting point of the study of stability of functional equations seems to be the famous talk of Ulam [21], in which he discussed a number of important unsolved problems. Among those was the question concerning the stability of group homomorphisms:

Let $G_{1}$ be a group and let $G_{2}$ be a metric group with a metric $d(\cdot, \cdot)$. Given $\varepsilon>0$, does there exist a $\delta>0$ such that if a function $h: G_{1} \rightarrow G_{2}$ satisfies the inequality $d(h(x y), h(x) h(y))<\delta$ for all $x, y \in G_{1}$, then there exists $a$ homomorphism $H: G_{1} \rightarrow G_{2}$ with $d(h(x), H(x))<\varepsilon$ for all $x \in G_{1}$ ?

The case of approximately additive functions was solved by Hyers [6] under the assumption that $G_{1}$ and $G_{2}$ are Banach spaces. Later, the result of Hyers was generalized by Rassias [14] and other mathematicians (see [3, 4, 5, 7, 8, 9, 10, 11, 12, 15, 16, 17, 19, 20]). The work of Rassias stimulated a number of mathematicians to investigate the stability problem of various functional equations. The terminology "Hyers-UlamRassias stability" of the additive Cauchy equation was originated from these historical backgrounds. This terminology is also applied to the other functional equations.

For a given integer $m \geq 2$, let $\left(a_{i j}\right)$ be a matrix in $\mathbb{C}^{m \times m}$ whose determinant $\omega$ is not zero. For $i \in\{1, \ldots, m\}$, we denote by $\omega_{i}$ the determinant of the matrix that remains after all entries of the $i$ th column in $\left(a_{i j}\right)$ are replaced by 1 .

In [13], the first author investigated the Hyers-Ulam-Rassias stability problem of a generalized additive Cauchy equation

$$
f\left(a_{1} x_{1}+\cdots+a_{m} x_{m}+x_{0}\right)=\sum_{i=1}^{m} b_{i} f\left(a_{i 1} x_{1}+\cdots+a_{i m} x_{m}\right)
$$

in the class of functions from a complex normed space $E_{1}$ into a complex Banach space $E_{2}$, where $a_{1}, \ldots, a_{m}, b_{1}, \ldots, b_{m}$ are complex numbers for which there exist some $j, k \in\{1, \ldots, m\}$ with $a_{j} \neq 0$ and $b_{k} \neq 0$. 
Let us define

$$
r:=\sum_{i=1}^{m} a_{i} \frac{\omega_{i}}{\omega}, \quad B:=\sum_{i=1}^{m} b_{i} .
$$

Given $x_{0} \in E_{1}$, we define a sequence $\left\{s_{n}(x)\right\}$ by

$$
s_{0}(x):=x, \quad s_{1}(x):=r x+x_{0}, \quad s_{n+1}(x):=s_{n}\left(s_{1}(x)\right)
$$

for any $x \in E_{1}$ and $n \in \mathbb{N}$. Assume that a function $\varphi: E_{1}^{m} \rightarrow[0, \infty)$ satisfies

$$
\begin{gathered}
\Phi\left(x_{1}, \ldots, x_{m}\right):=\sum_{i=0}^{\infty} \frac{1}{|B|^{i+1}} \varphi\left(\frac{\omega_{1}}{\omega} s_{i}\left(x_{1}\right), \ldots, \frac{\omega_{m}}{\omega} s_{i}\left(x_{m}\right)\right)<\infty, \\
\Phi\left(s_{n}(x), \ldots, s_{n}(x)\right)=o\left(|B|^{n}\right) \quad \text { as } n \longrightarrow \infty
\end{gathered}
$$

for all $x, x_{1}, \ldots, x_{m} \in E_{1}$.

The following is one of the main theorems in [13].

THEOREM 1.1. Let $E_{1}$ and $E_{2}$ be a complex normed space and a complex Banach space, respectively. Assume that a function $f: E_{1} \rightarrow E_{2}$ satisfies the inequality

$$
\left\|f\left(a_{1} x_{1}+\cdots+a_{m} x_{m}+x_{0}\right)-\sum_{i=1}^{m} b_{i} f\left(a_{i 1} x_{1}+\cdots+a_{i m} x_{m}\right)\right\| \leq \varphi\left(x_{1}, \ldots, x_{m}\right)
$$

for all $x_{1}, \ldots, x_{m} \in E_{1}$ and for a fixed $x_{0} \in E_{1}$. If $|r|,|B| \notin\{0,1\}$, then there exists $a$ unique function $F: E_{1} \rightarrow E_{2}$ such that

$$
F\left(r x+x_{0}\right)=B F(x), \quad\|f(x)-F(x)\| \leq \Phi(x, \ldots, x)
$$

for all $x \in E_{1}$.

The following familiar functional equation

$$
f(a x+b)=\operatorname{cf}(x)
$$

frequently appear in solving various functional equations (especially, in solving the generalized additive Cauchy equations). But to the best of our knowledge no author solved this equation. In Section 2, we solve the functional equation (1.8) in the class of functions $f: \mathbb{R} \rightarrow \mathbb{R}$. And, in Section 3, we use the result of Section 2 to study the Hyers-Ulam-Rassias stability of the generalized additive Cauchy equation (1.1). The question of Rassias and Tabor concerning the stability of the generalized additive Cauchy equations is studied in Section 4.

2. General solution of (1.8). Aczél and Kuczma, [1], solved a familiar functional equation

$$
f(k x)=k^{\gamma} f(x),
$$

where $\gamma$ is a real constant and $k \neq 1$ is a positive constant. Indeed, they proved the following theorem. 
THEOREM 2.1. The general solution $f:(0, \infty) \rightarrow \mathbb{R}$ of (2.1) is given by

$$
f(x)=x^{\gamma} p\left(\log _{k} x\right),
$$

where $p: \mathbb{R} \rightarrow \mathbb{R}$ is an arbitrary periodic function of period 1 .

By using the idea of Aczél and Kuczma, we solve (1.8).

THEOREM 2.2. Let $a>0(a \neq 1), b$ and $c>0$ be real constants. The general solution $f: \mathbb{R} \rightarrow \mathbb{R}$ of the functional equation (1.8) is given by

$$
f(x)= \begin{cases}c^{\log _{a}(x+b /(a-1))} p_{1}\left[\log _{a}\left(x+\frac{b}{a-1}\right)\right] & \left(x>-\frac{b}{a-1}\right), \\ d & \left(x=-\frac{b}{a-1}\right), \\ c^{\log _{a}(-x-b /(a-1))} p_{2}\left[\log _{a}\left(-x-\frac{b}{a-1}\right)\right] & \left(x<-\frac{b}{a-1}\right),\end{cases}
$$

where $p_{1}, p_{2}: \mathbb{R} \rightarrow \mathbb{R}$ are arbitrary periodic functions of period 1 . If $c \neq 1, d=0$ and if $c=1, d$ is an arbitrary real number.

Proof. Assume that a function $f: \mathbb{R} \rightarrow \mathbb{R}$ is given by the formula (2.3). Obviously, $x>-b /(a-1), x=-b /(a-1)$, and $x<-b /(a-1)$ imply $a x+b>-b /(a-1)$, $a x+b=-b /(a-1)$, and $a x+b<-b /(a-1)$, respectively. The following facts

$$
\begin{aligned}
f(a x+b) & =c^{\log _{a}(a x+b+b /(a-1))} p_{1}\left[\log _{a}\left(a x+b+\frac{b}{a-1}\right)\right] \\
& =c^{\log _{a}(x+b /(a-1))+1} p_{1}\left[\log _{a}\left(x+\frac{b}{a-1}\right)+1\right] \\
& =c f(x)
\end{aligned}
$$

for all $x>-b /(a-1)$, and

$$
\begin{aligned}
f(a x+b) & =c^{\log _{a}(-a x-b-b /(a-1))} p_{2}\left[\log _{a}\left(-a x-b-\frac{b}{a-1}\right)\right] \\
& =c^{\log _{a}(-x-b /(a-1))+1} p_{2}\left[\log _{a}\left(-x-\frac{b}{a-1}\right)+1\right] \\
& =c f(x)
\end{aligned}
$$

for any $x<-b /(a-1)$, together with

$$
f(a x+b)=f\left(-\frac{b}{a-1}\right)=c f(x)
$$

for $x=-b /(a-1)$, imply that $f$ is a solution of (1.8).

Now, assume that $f: \mathbb{R} \rightarrow \mathbb{R}$ is a solution of equation (1.8). Let us define functions $p_{1}, p_{2}: \mathbb{R} \rightarrow \mathbb{R}$ by

$$
p_{1}(t):=c^{-t} f\left(a^{t}-\frac{b}{a-1}\right), \quad p_{2}(t):=c^{-t} f\left(-a^{t}-\frac{b}{a-1}\right) .
$$


Then,

$$
\begin{aligned}
p_{1}(t+1) & =c^{-t-1} f\left(a^{t+1}-\frac{b}{a-1}\right)=c^{-t-1} f\left[a\left(a^{t}-\frac{b}{a-1}\right)+b\right] \\
& =c^{-t} f\left(a^{t}-\frac{b}{a-1}\right)=p_{1}(t), \\
p_{2}(t+1) & =c^{-t-1} f\left(-a^{t+1}-\frac{b}{a-1}\right)=c^{-t-1} f\left[a\left(-a^{t}-\frac{b}{a-1}\right)+b\right] \\
& =c^{-t} f\left(-a^{t}-\frac{b}{a-1}\right)=p_{2}(t)
\end{aligned}
$$

which mean that $p_{1}$ and $p_{2}$ are periodic functions of period 1 .

If we put $t=\log _{a}(x+b /(a-1))$ and $t=\log _{a}(-x-b /(a-1))$ in the first and second definition of (2.7), respectively, then we see that (2.3) is true.

3. Hyers-Ulam-Rassias stability of (1.1). Throughout this section, let $\left(a_{i j}\right)$ be a matrix in $\mathbb{R}^{m \times m}$ whose determinant $\omega$ is nonzero, where $m$ is an integer greater than 1 . We denote by $\omega_{i}$ the determinant of the matrix that remains after all entries of the $i$ th column in $\left(a_{i j}\right)$ are replaced by 1 . Let $a_{i}$ and $b_{i}, i \in\{1, \ldots, m\}$, be real numbers for which there exist some $j, k \in\{1, \ldots, m\}$ with $a_{j} \neq 0$ and $b_{k} \neq 0$. Let a function $\varphi: E_{1}^{m} \rightarrow[0, \infty)$ satisfy the conditions (1.5) and (3.1), instead of (1.4), for all $x \in E_{1}$ :

$$
\Phi(x, \ldots, x):=\sum_{i=0}^{\infty} \frac{1}{|B|^{i+1}} \varphi\left(\frac{\omega_{1}}{\omega} s_{i}(x), \ldots, \frac{\omega_{m}}{\omega} s_{i}(x)\right)<\infty .
$$

The following lemma is a reduced version of Theorem 1.1 which will be applied to the case of real normed spaces. We may slightly modify the proof of Theorem 1.1 to prove the following lemma.

LEMMA 3.1. Let $E_{1}$ be a real normed space and $E_{2}$ a real Banach space. If a function $f: E_{1} \rightarrow E_{2}$ satisfies inequality (1.6) for all $x_{1}, \ldots, x_{m} \in E_{1}$ and for some $x_{0} \in E_{1}$ and $|B|>0$, then there exists a unique function $F: E_{1} \rightarrow E_{2}$ such that

$$
F\left(r x+x_{0}\right)=B F(x), \quad\|f(x)-F(x)\| \leq \Phi(x, \ldots, x)
$$

for each $x \in E_{1}$.

Combining Theorem 2.2 and Lemma 3.1, we obtain the following theorem.

THEOREM 3.2. Assume that a function $f: \mathbb{R} \rightarrow \mathbb{R}$ satisfies the inequality (1.6) for all $x_{1}, \ldots, x_{m} \in \mathbb{R}$ and for some $x_{0} \in \mathbb{R}$. Let $r$ and $B$ be given by the formulae in (1.2). If $r>0(r \neq 1)$ and $B>0$, then there exists a unique function $F: \mathbb{R} \rightarrow \mathbb{R}$ such that

$$
\|f(x)-F(x)\| \leq \Phi(x, \ldots, x)
$$


for all $x \in \mathbb{R}$. Furthermore, the function $F$ may be represented by

$$
F(x)= \begin{cases}B^{\log _{r}\left(x+x_{0} /(r-1)\right)} p_{1}\left[\log _{r}\left(x+\frac{x_{0}}{r-1}\right)\right] & \left(x>-\frac{x_{0}}{r-1}\right), \\ d & \left(x=-\frac{x_{0}}{r-1}\right), \\ B^{\log _{r}\left(-x-x_{0} /(r-1)\right)} p_{2}\left[\log _{r}\left(-x-\frac{x_{0}}{r-1}\right)\right] & \left(x<-\frac{x_{0}}{r-1}\right),\end{cases}
$$

where $p_{1}, p_{2}: \mathbb{R} \rightarrow \mathbb{R}$ are periodic functions of period 1 . If $B \neq 1, d=0$ and if $B=1, d$ is an arbitrary real number.

4. Question of Rassias and Tabor. Rassias and Tabor [18] asked whether the generalized Cauchy functional equation

$$
f\left(a_{1} x+a_{2} y+v\right)=b_{1} f(x)+b_{2} f(y)+w,
$$

where $a_{1} a_{2} b_{1} b_{2} \neq 0$, is stable in the sense of Hyers, Ulam, and Rassias. For the case when $a_{1}=b_{1}, a_{2}=b_{2}$, and $v=w=0$ in (4.1), Badea [2] proved the Hyers-UlamRassias stability. Furthermore, the stability problem for the case of $v \neq 0$ in (4.1) was investigated in [13].

We will give a partial answer to the question of Rassias and Tabor which is indeed a corollary to Theorem 3.2.

THEOREM 4.1. Given real numbers $a, b>0$, let $a_{1}, a_{2}, b_{1}, b_{2}$ be real constants with $a_{1}+a_{2}>0, a_{1}+a_{2} \neq 1, b_{1}+b_{2}>\max \left\{\left(a_{1}+a_{2}\right)^{a},\left(a_{1}+a_{2}\right)^{b}\right\}$, and $b_{1}+b_{2} \neq 1$. If $a$ function $f: \mathbb{R} \rightarrow \mathbb{R}$ satisfies the inequality

$$
\left|f\left(a_{1} x+a_{2} y\right)-b_{1} f(x)-b_{2} f(y)-w\right| \leq \theta\left(|x|^{a}+|y|^{b}\right)
$$

for some $\theta \geq 0$, for a fixed $w \in \mathbb{R}$ and for all $x, y \in \mathbb{R}$, then there exists a unique function $F: \mathbb{R} \rightarrow \mathbb{R}$ with

$$
\left|f(x)-F(x)-\frac{w}{1-b_{1}-b_{2}}\right| \leq \frac{\theta|x|^{a}}{b_{1}+b_{2}-\left(a_{1}+a_{2}\right)^{a}}+\frac{\theta|x|^{b}}{b_{1}+b_{2}-\left(a_{1}+a_{2}\right)^{b}}
$$

for any $x \in \mathbb{R}$, where

$$
F(x)= \begin{cases}\left(b_{1}+b_{2}\right)^{\log _{a_{1}+a_{2}} x} p_{1}\left(\log _{a_{1}+a_{2}} x\right) & (x>0), \\ 0 & (x=0), \\ \left(b_{1}+b_{2}\right)^{\log _{a_{1}+a_{2}}(-x)} p_{2}\left(\log _{a_{1}+a_{2}}(-x)\right) & (x<0),\end{cases}
$$

with periodic functions $p_{1}, p_{2}: \mathbb{R} \rightarrow \mathbb{R}$ of period 1 .

Proof. With $g(x)=f(x)-w /\left(1-b_{1}-b_{2}\right)$, inequality (4.2) yields

$$
\left|g\left(a_{1} x+a_{2} y\right)-b_{1} g(x)-b_{2} g(y)\right| \leq \theta\left(|x|^{a}+|y|^{b}\right)
$$


for all $x, y \in \mathbb{R}$. For this case, we have $a_{11}=1, a_{12}=0, a_{21}=0, a_{22}=1$, and so $\omega=\omega_{1}=\omega_{2}=1$. If we put $\varphi(x, y)=\theta\left(|x|^{a}+|y|^{b}\right)$, then $\varphi: \mathbb{R} \times \mathbb{R} \rightarrow[0, \infty)$ satisfies conditions (1.5) and (3.1). Hence, Lemma 3.1 says that there exists a unique function $F: \mathbb{R} \rightarrow \mathbb{R}$ such that

$$
\begin{aligned}
& F\left(\left(a_{1}+a_{2}\right) x\right)=\left(b_{1}+b_{2}\right) F(x), \\
|g(x)-F(x)| \leq & \Phi(x, x) \\
= & \sum_{i=0}^{\infty} \frac{\theta}{\left(b_{1}+b_{2}\right)^{i+1}}\left(\left|\left(a_{1}+a_{2}\right)^{i} x\right|^{a}+\left|\left(a_{1}+a_{2}\right)^{i} x\right|^{b}\right) \\
= & \frac{\theta|x|^{a}}{b_{1}+b_{2}-\left(a_{1}+a_{2}\right)^{a}}+\frac{\theta|x|^{b}}{b_{1}+b_{2}-\left(a_{1}+a_{2}\right)^{b}}
\end{aligned}
$$

for all $x \in \mathbb{R}$.

Theorem 2.2, together with (4.6), implies (4.4).

\section{REFERENCES}

[1] J. Aczél and M. Kuczma, Solutions of a functional equation, convex of higher order, General Inequalities, 6 (Oberwolfach, 1990) (Basel), Internat. Ser. Numer. Math., vol. 103, Birkhäuser, 1992, pp. 209-213. MR 94d:39007. Zbl 772.39002.

[2] C. Badea, On the Hyers-Ulam stability of mappings: the direct method, Stability of Mappings of Hyers-Ulam Type (Palm Harbor, FL), Hadronic Press Collect. Orig. Artic., Hadronic Press, 1994, pp. 7-13. MR 96b:39022. Zbl 845.39011.

[3] G. L. Forti, Hyers-Ulam stability of functional equations in several variables, Aequationes Math. 50 (1995), no. 1-2, 143-190. MR 96i:39033. Zbl 836.39007.

[4] Z. Gajda, On stability of additive mappings, Int. J. Math. Math. Sci. 14 (1991), no. 3, 431434. MR 92e:39029. Zbl 739.39013.

[5] P. Găvruța, A generalization of the Hyers-Ulam-Rassias stability of approximately additive mappings, J. Math. Anal. Appl. 184 (1994), no. 3, 431-436. MR 95e:47089. Zbl 818.46043.

[6] D. H. Hyers, On the stability of the linear functional equation, Proc. Nat. Acad. Sci. U.S.A. 27 (1941), 222-224. MR 2,315a. Zbl 061.26403.

[7] D. H. Hyers, G. Isac, and T. M. Rassias, On the asymptoticity aspect of Hyers-Ulam stability of mappings, Proc. Amer. Math. Soc. 126 (1998), no. 2, 425-430. MR 98d:39004. Zbl 894.39012.

[8] _ Stability of Functional Equations in Several Variables, Progress in Nonlinear Differential Equations and their Applications, vol. 34, Birkhäuser Boston Inc., Boston, MA, 1998. MR 99i:39035. Zbl 907.39025.

[9] D. H. Hyers and T. M. Rassias, Approximate homomorphisms, Aequationes Math. 44 (1992), no. 2-3, 125-153. MR 93i:39007. Zbl 806.47056.

[10] S.-M. Jung, Hyers-Ulam-Rassias Stability of Functional Equations in Mathematical Analysis, Hadronic Press, to appear.

[11] _ On the Hyers-Ulam-Rassias stability of approximately additive mappings, J. Math. Anal. Appl. 204 (1996), no. 1, 221-226. MR 97m:47105. Zbl 888.46018.

[12] _ Hyers-Ulam-Rassias stability of functional equations, Dynam. Systems Appl. 6 (1997), no. 4, 541-565. MR 98m:39067. Zbl 891.39025.

[13] __ On modified Hyers-Ulam-Rassias stability of a generalized Cauchy functional equation, Nonlinear Stud. 5 (1998), no. 1, 59-67. MR 99h:39030. Zbl 907.39029.

[14] T. M. Rassias, On the stability of the linear mapping in Banach spaces, Proc. Amer. Math. Soc. 72 (1978), no. 2, 297-300. MR 80d:47094. Zbl 398.47040. 

, The stability of mappings and related topics, Aequ. Math. 39 (1990), 292-293, Report of the 27 th ISFE.

[16] _ On a modified Hyers-Ulam sequence, J. Math. Anal. Appl. 158 (1991), no. 1, 106113. MR 92d:46046. Zbl 746.46038.

[17] T. M. Rassias and P. Šemrl, On the behavior of mappings which do not satisfy HyersUlam stability, Proc. Amer. Math. Soc. 114 (1992), no. 4, 989-993. MR 92g:47101. Zbl 761.47004.

[18] T. M. Rassias and J. Tabor, What is left of Hyers-Ulam stability?, J. Natur. Geom. 1 (1992), no. 2, 65-69. MR 93b:47131. Zbl 757.47032.

[19] _ On approximately additive mappings in Banach spaces, Stability of Mappings of Hyers-Ulam Type (Palm Harbor, FL), Hadronic Press Collect. Orig. Artic., Hadronic Press, 1994, pp. 127-134. MR 95k:39029. Zbl 845.39013.

[20] T. M. Rassias and P. Šemrl, On the Hyers-Ulam stability of linear mappings, J. Math. Anal. Appl. 173 (1993), no. 2, 325-338. MR 94d:39011. Zbl 789.46037.

[21] S. M. Ulam, Problems in Modern Mathematics, Science Editions John Wiley \& Sons, Inc., New York, 1964. MR 43\#6031. Zbl 137.24201.

Soon-Mo Jung: Mathematics Section, College of Science And Technology, Hong-IK UNIVERSITY, CHOCHIWON 339-701, KOREA

E-mail address: smjung@wow.hongik.ac.kr

Ki-SuK LeE: Department of MAthematics EducAtion, Korea NATional University of EDUCATION, CHONGWON 363-791, CHOONGBOOK, KOREA

E-mail address: kslee@cc. knue.ac.kr 


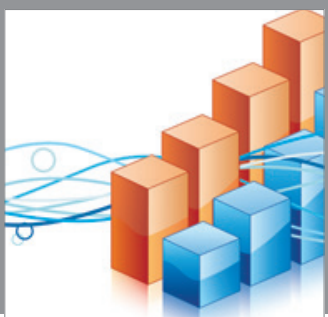

Advances in

Operations Research

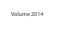

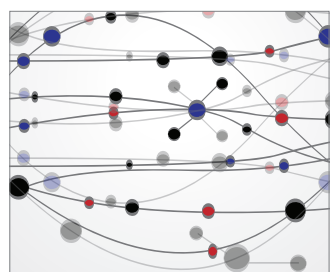

\section{The Scientific} World Journal
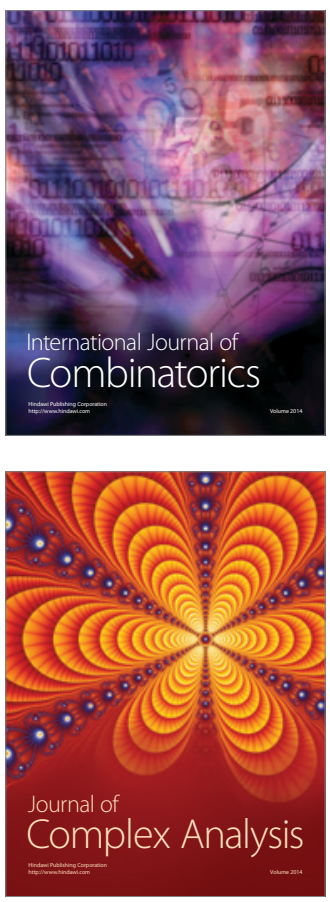

International Journal of

Mathematics and

Mathematical

Sciences
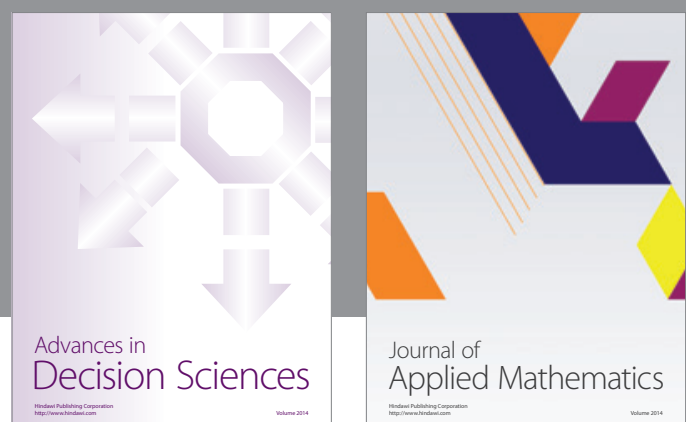

Journal of

Applied Mathematics
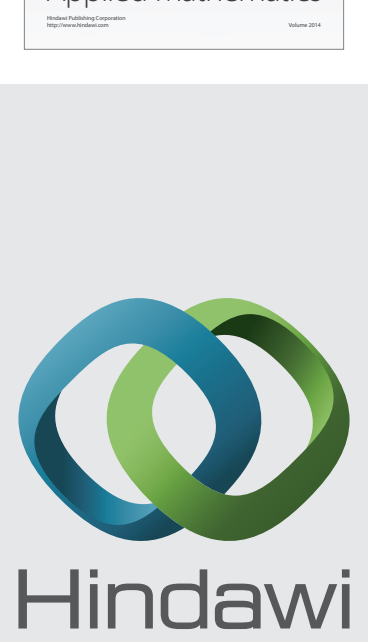

Submit your manuscripts at http://www.hindawi.com
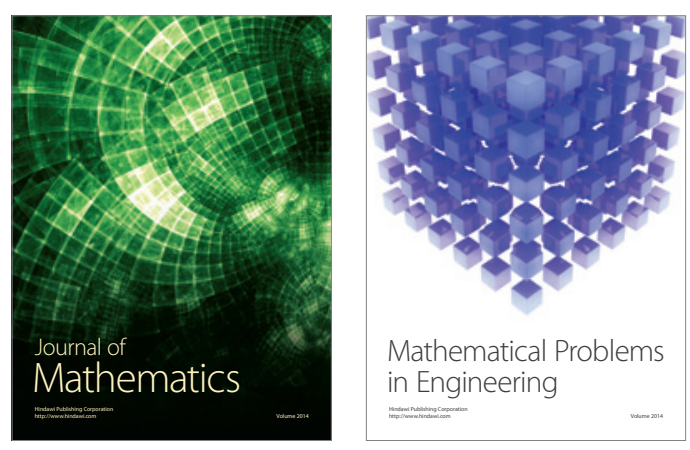

Mathematical Problems in Engineering
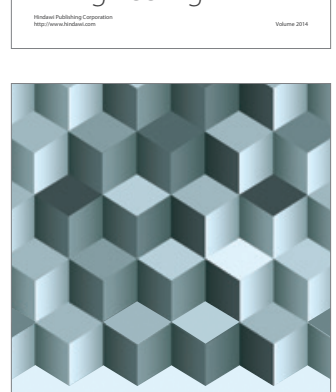

Journal of

Function Spaces
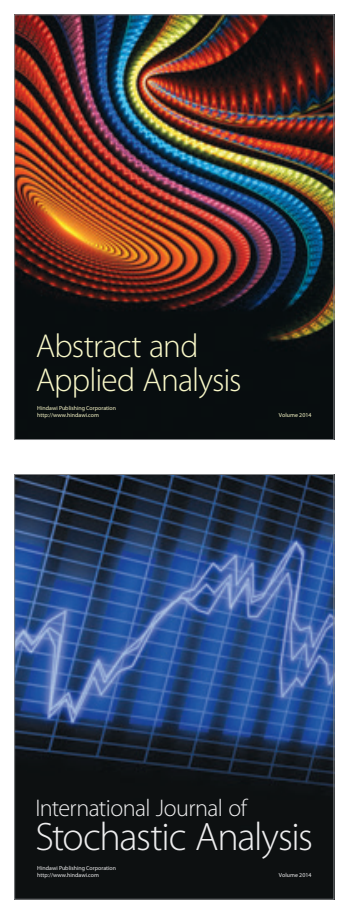

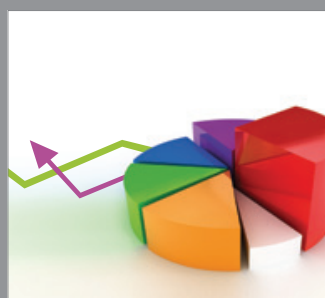

ournal of

Probability and Statistics

Promensencen
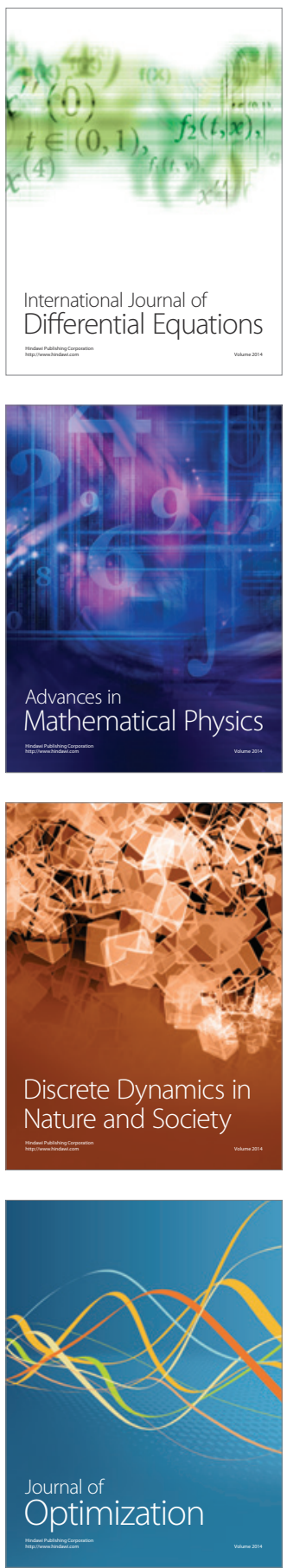